\title{
Atomic Scale Self Accommodation Observed at hcp/dhcp Martensitic Transformation Interfaces
}

\author{
T. Waitz, H.P. Karnthaler and C. Rentenberger \\ Institut für Material physik, University of Vienna, Boltzmanngasse 5, 1090 Vienna, Austria
}

\begin{abstract}
The martensitic hcp $\rightarrow$ dhcp transformation was studied in single crystals of Co- 0.85 at.\% $\mathrm{Fe}$ by transmission electron microscopy (TEM) methods. During in-situ heating dhcp lamellae emerge into the hcp areas of the TEM specimens. The minimum thickness of the lamellae is about 15 close packed planes. Frequently lamellae are observed that grow in thickness by the movement of superledges that are up to about 100 close packed planes in height. The partials at the fronts of the transformation were analysed on an atomic level in TEM images with atomic resolution. The analysis shows that the transformation fronts consists of a sequence of very narrow dipoles compensating their long range strain fields. Since dipoles of a common Burgers vector are observed it is proposed that their short range interactions might promote their correlated nucleation.
\end{abstract}

\section{INTRODUCTION}

In pure $\mathrm{Co}, \mathrm{CoNi}$ and $\mathrm{Fe}$ based shape memory alloys martensitic phase transformations take place between the face centred cubic (fcc) high temperature phase and the hexagonal close packed (hcp) structure [1]. In CoFe alloys a third, intermediate phase occurs that is double hexagonal close packed (dhcp) [2]. The interatomic distances of the three phases are nearly the same. In fig. I the phase diagram of CoFe on the Co rich side is illustrated showing the dhcp phase region (based on the data given in [3]). The solid and broken lines represent the fcc $\leftrightarrow$ dhcp and dhcp $\leftrightarrow$ hcp transformations, respectively; the hysteresis between heating and cooling is indicated by the curves $h$ and $c$. It seems that the hcp phase occurs only up to an iron content of about 1.5 at.\%.

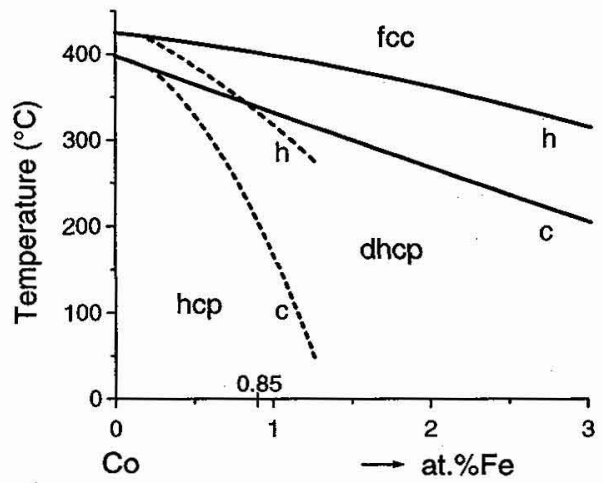

Figure 1: Schematic phase diagram of the CoFe system on the Co rich side. The curves $h$ and $\mathrm{c}$ indicate the hysteresis between heating and cooling.

Transformations between the close packed lattices (fcc, dhcp and hcp) can be achieved by the movement of glissile transformation dislocations (called partials in the following). The partials can change the structure by altering both the stacking sequence and the spacing of the close packed (0001) planes since 
they have a Shockley partial Burgers vector $\mathbf{p}_{\mathrm{i}}$ and in addition, they can contain a small Burgers vector component perpendicular to the close packed planes [4]. It is important to mention that a glissile phase boundary (transformation front) can be formed by the correlated movement of the partials. When they are correlated on an atomic scale two different modes of the transformation can be distinguished [5]. Firstly, mode A: all the partials at the transformation front have the same Burgers vector. Secondly, mode B: the transformation front contains dislocations with different Burgers vectors that compensate each other on an atomic scale.

It is the purpose of the present investigation to image the atomic structures that occur at the hcp/dhcp transformation fronts in Co-0.85at.\% Fe single crystals. The Burgers vectors of the partials can be analysed quantitatively by drawing Burgers circuits around them in high resolution transmission electron microscopy (HRTEM) images. This allows in a direct way to determine the appropriate atomistic model.

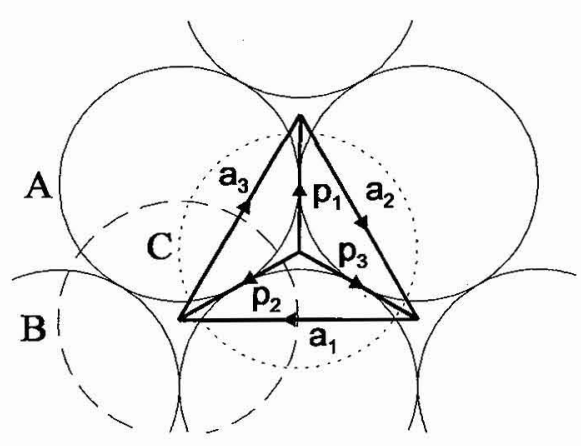

(a)

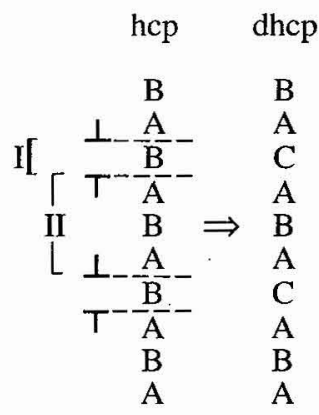

(b)

Figure 2: (a) Sketch of the close packed layers projected onto the (0001) plane. Atoms of the A, B and C layers are indicated by solid, broken and dotted lines, respectively. The burgers vectors $\mathbf{p}_{i}$ and $\mathbf{a}_{i}$ indicate the partial and total dislocations. (b) The hcp $\rightarrow$ dhcp transformation: partials gliding above and below every other B layer change the AB (hcp) stacking sequence into $\mathrm{ABAC}$ (dhcp). Partials represented by the symbol $\perp$ and $\mathrm{T}$ can have any one of the Burgers vectors $+\mathbf{p}_{\mathrm{i}}$ and $-\mathbf{p}_{\mathrm{i}}$, respectively. Two different types of dipoles are occurring, they are marked I and II.

\section{EXPERIMENTAL PROCEDURE}

A CoFe alloy with a nominal composition $\mathrm{Co}-0.85$ at.\% was made of high purity components (Co and $\mathrm{Fe}$; 4N8 from Johnson Matthey Ltd). A polycrystalline rod was used to grow a single crystal of the fcc phase by a modified Bridgman method in a "soft" mould of sintered $\mathrm{Al}_{2} \mathrm{O}_{3}$ powder under vacuum $\left(<10^{-3} \mathrm{~Pa}\right)$ at a speed of $\sim 15 \mathrm{~mm} / \mathrm{h}$. The temperature of the furnace was kept above $500^{\circ} \mathrm{C}$; therefore the crystal remained in the fcc high temperature phase. Immediately afterwards the crystal was cyclically annealed for about $24 \mathrm{~h}$ at $1350^{\circ} \mathrm{C}$ to reduce the density of grown-in defects. In a second step the temperature of the furnace was lowered to $500^{\circ} \mathrm{C}$, and the fcc $\rightarrow$ dhcp $\rightarrow$ hcp transformation was achieved by moving the crystal out of the hot zone of the furnace at a speed of $50 \mathrm{~mm} / \mathrm{h}$. X-ray analysis showed that the crystal was hcp at RT. Two out of the four possible $\{111\}$ hcp variants were present, and the maximum length of a single-variant hcp crystal was about $50 \mathrm{~mm}$.

For the TEM investigations it is necessary to minimise the aberrations caused by the ferromagnetic specimens by keeping their mass small. Tiny discs (only about $1 \mathrm{~mm}$ in diameter and about $0.15 \mathrm{~mm}$ thick) were prepared with $a<1 \overline{1} 20>$ orientation by spark erosion from a single-variant hcp crystal. The discs were used to prepare the TEM foils by jet polishing and final bench polishing both done at RT. The specimens were transformed by in-situ heating during the observation in the electron microscope (Philips EM300 operating at $100 \mathrm{kV}$ ). After the in-situ experiments the specimens were cooled to room temperature (RT) and were used to carry out HRTEM investigations at $300 \mathrm{kV}$ (Philips CM 30 ST TEM instrument). In addition; bulk specimens were transformed to dhcp by heating them to $400^{\circ} \mathrm{C}$; afterwards their electropolished surfaces were investigated at RT by light optical microscopy methods.

\section{STRUCTURAL ASPECTS OF THE hcp $\rightarrow$ dhcp TRANSFORMATION}

Fig. $2 \mathrm{a}$ is a sketch of the close packed layers showing the three Shockley partial Burgers vectors $\mathbf{p}_{\mathrm{i}}$ (of the type $1 / 3<1 \overline{1} 00>$ ). A pair of partials (on neighbouring planes) gliding on every fourth close packed plane 


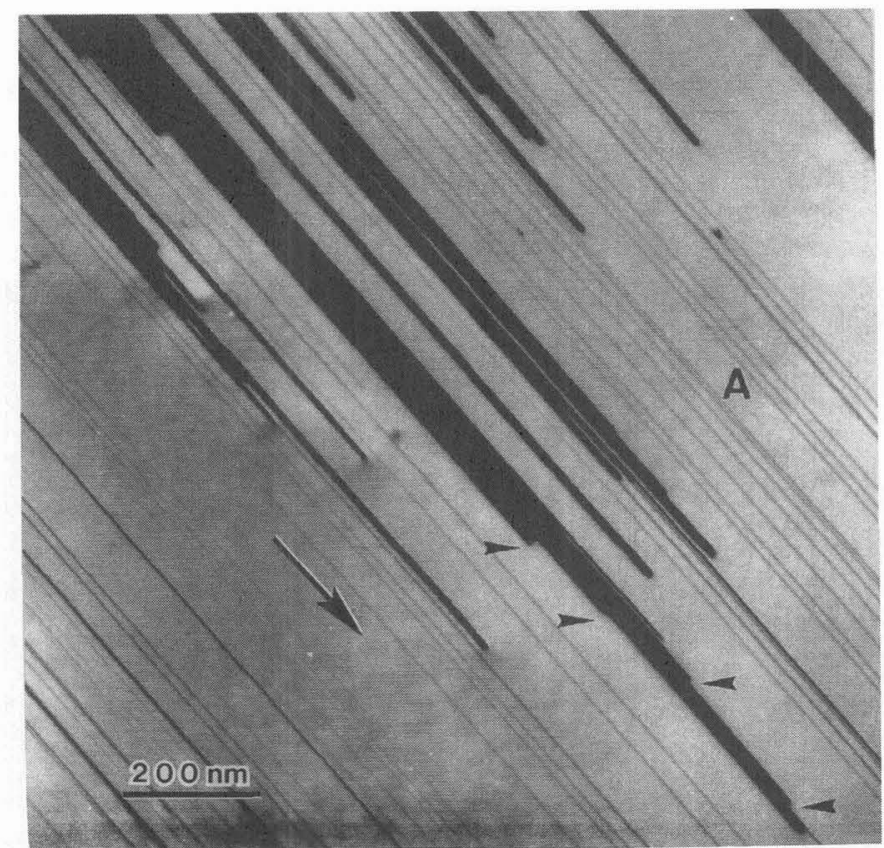

Figure 3: TEM bright field image of the hcp $\rightarrow$ dhcp phase transformation in a Co- 0.85 at.\%Fe single crystal during in-situ heating. Dhcp lamellae (showing dark contrast) emerge from the bulk areas towards the thin areas of the TEM foil. (The arrow indicates the direction in which the transformation fronts move.) Some superledges are indicated by smaller arrows. The fine lines (e.g. near A) correspond to stacking faults that existed in the hep matrix prior the transformation.

(cf. fig. 2b) can change the $\mathrm{AB}$ (hcp) stacking sequence into $\mathrm{ABAC}$ (dhcp) [6]. In fig. 2b the partials indicated by the symbol $\perp$ can have any one of the three Burgers vectors $+\mathbf{p}_{\mathbf{i}}$; whereas, partials indicated by the symbol $T$ can have any one of the three Burgers vectors $-p_{i}$. The two partials gliding on neighbouring planes can either attract or repel each other. In the first case partials of opposite Burgers vectors form a dipole: $\mathbf{p}_{\mathrm{i}}-\mathbf{p}_{\mathrm{i}}=\mathbf{0}$; in the model of fig. $2 \mathrm{~b}$ two types of dipoles can be distinguished (termed type $I$ and type II dipoles) [6]. In the second case partials $\mathbf{p}_{\mathbf{i}},-\mathbf{p}_{\mathbf{j}}(\mathbf{i} \neq \mathbf{j})$ sum up to a Burgers vector equal to that of a total dislocation (of the type $1 / 3<11 \overline{2} 0>$ ) e.g. $\mathbf{p}_{1}-\mathbf{p}_{2}=\mathbf{a}_{3}$. (It should be noted that the partials can not combine to a total dislocation since they are gliding on different planes).

\section{EXPERIMENTAL RESULTS}

\subsection{The transformation hcp $\rightarrow$ dhcp achieved by in-situ heating}

Fig. 3 shows a TEM bright field image of a hcp specimen partially transformed to dhcp during in-situ heating. Dhcp lamellae showing dark contrast emerge from the bulk areas of the TEM specimen and therefore their nucleation could not be observed. The first lamellae moving into the thin area of the specimen were detected at about $430^{\circ} \mathrm{C}$ (caused by the influence of a surface friction in the thin TEM foil this is higher than the austenite start temperature in the bulk (cf. fig. 1) [7]) The minimum thickness of the lamellae is about $3 \mathrm{~nm}$ (15 atomic planes). Frequently, lamellae are observed that grow in thickness by the movement of superledges (indicated by arrows in fig. 3) which are up to $\sim 25 \mathrm{~nm}$ in height and contain about 50 partials. The formation of individual stacking faults was not observed during in-situ heating.

Caused by the friction effect the dhcp lamellae did not retreat during cooling to RT. Therefore it was possible to transfer the specimens without structural changes to a HRTEM instrument and to analyse the structures at the transformation fronts in detail at RT.

\subsection{TEM images of the transformation front}

Fig. 4 shows a TEM lattice fringe image of a specimen transformed by in-situ heating. The dhcp transformation fronts containing the partials are indicated by arrows. There is a distinct change of a 4 
layer fringe contrast of the dhcp lattice to a 2 layer fringe contrast of the retained hcp phase. The interfaces containing the partials are straight and almost perpendicular to both the coherent (0001) interface and the surface of the thin TEM foil. No indication of local strains occurring at the transformation fronts was obtained using different TEM methods.

Fig. 5 is a HRTEM image of a terminating dhcp lamella. The atomic columns of both the hcp and dhcp lattice are resolved. The defocus conditions were selected in such way that the contrast of the atomic columns corresponds to the white dots. The transformation front (indicated by two arrows) contains 26 partials. A circuit drawn around the interface containing all the partials shows no closure failure.

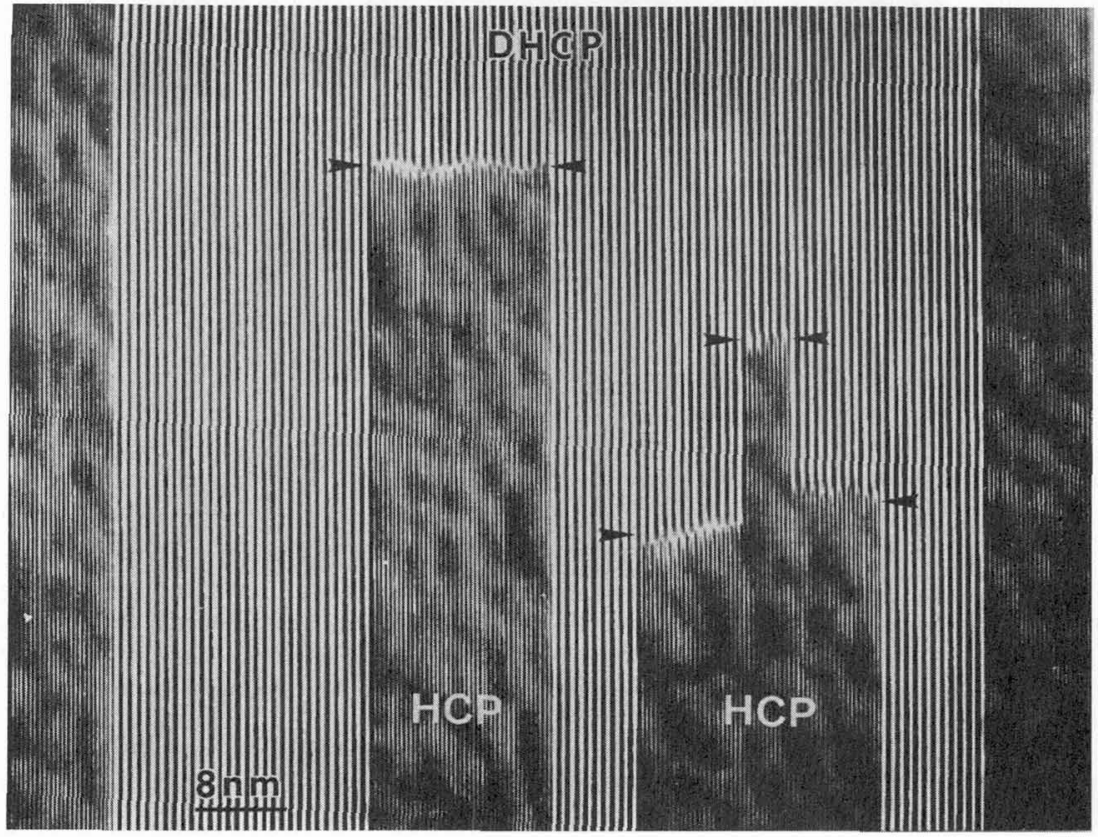

Figure 4: TEM lattice fringe image of the transformation fronts. The periodicity of the fringes is 2 and 4 close packed layers in the hcp and dhcp phase, respectively. The interfaces containing the partials (indicated by arrows) are straight and almost perpendicular to both the coherent (0001) habit plane and the surface of the TEM foil. Strain contrast is not observed at the transformation fronts.

\subsection{Analysis of the partials at the transformation fronts by circuits}

To analyse the partials at the transformation fronts circuits drawn around them in HRTEM images can be used. However, only the component of the Burgers vector (edge component) perpendicular to the plane of the TEM foil can be analysed [7]. The edge component of the total Burgers vector is zero in fig. 5 since the circuit drawn around all the partials shows no closure failure. In addition, circuits were drawn around each individual partial (these circuits are not shown in fig. 5). The analysis yields that the Burgers vectors of partials on adjacent planes have opposite edge components. This result is in agreement with the transformation model based on partial dipoles since they have zero total Burgers vector (cf. fig. $2 b$ ). According to fig. $2 \mathrm{~b}$ the dipoles can have any one of three Burgers vectors $\pm \mathbf{p}_{\mathrm{i}}$. The analysis carried out in fig. 5 shows that out of the three $\pm \mathbf{p}_{\mathbf{i}}$ only one (e.g. $\left.\pm \mathbf{p}_{1}\right)$ occurs.

\section{DISCUSSION}

\subsection{Atomic scale self accommodation}

The results of the analysis of HRTEM images shows that the partials on adjacent layers are correlated on an atomic scale since they are forming dipoles. Only one out of the three possible Shockley partial Burgers vectors is observed in fig. 5 and therefore both type I and II dipoles occur. This result is in agreement with the absence of long range strain contrasts at the interfaces containing the partials since the narrow dipoles are compensating their long range strain fields almost completely. In addition, the shape of 


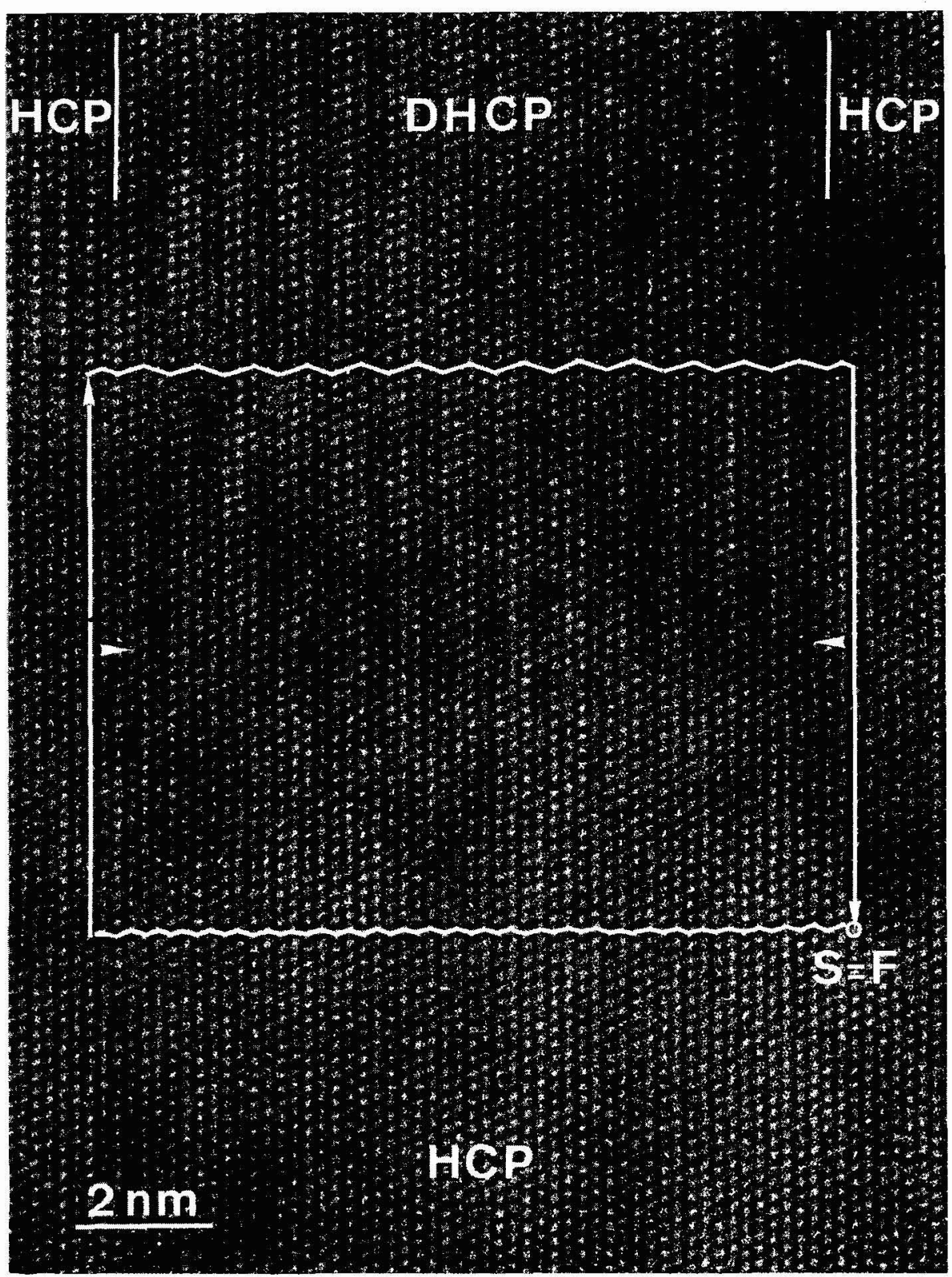

Figure 5: HRTEM image of a terminating dhcp lamella. The coherent (0001) interfaces are marked by two white lines whereas the interface containing the partials is indicated by two arrows. A circuit drawn around all the partials shows no closure failure.

the transformation fronts are planar which agrees with partials that are minimising their separations. Therefore it is concluded that all the partials at the interface are attracting each other. In the case of the $2 \mathrm{H}$ to $4 \mathrm{H}$ martensitic transformation in $\mathrm{TiCr}_{2}$ a curved interface containing partials was observed. It was concluded that the interface contains type I dipoles; however, in the HRTEM images individual atomic 
columns of the $4 \mathrm{H}$ structure were not resolved (caused by a slight misalignment of the TEM specimen) and an analysis by drawing circuits was not carried out.

The results show that the hcp to dhcp transformation occurs by lamellae that contain minimum coherency strains. The long range strain fields of the partials are accommodated by the narrow type I dipoles. Residual, short range elastic interactions might promote the nucleation of a lamella bounded by a repeated sequence of dipoles of a common Burgers vector causing the formation of dipoles of type II. In addition, the formation of correlated dipoles is energetically favourable since it decreases the surface to volume ratio of a dhcp lamella compared to the nucleation of individual stacking faults. Lattice coherency strains arising by the slight difference of the interatomic distance of the close packed planes in the two phases might limit the maximum thickness of the dhcp lamellae. These conclusions are in agreement with the experimental observations since the transformation occurs by lamellae and ledges that have a thickness between 3 and $25 \mathrm{~nm}$.

Finally it should be noted that the results obtained by the TEM investigations agree with the results obtained of specimens transformed in the bulk. In the latter case the transformation causes no relief structure on electropolished crystal surfaces in agreement with the conclusion that the transformation is self accommodating on the atomic scale.

\subsection{Comparison with $\mathrm{dhcp} \leftrightarrow$ fec and hep $\leftrightarrow$ fcc transformations}

In the present work the correlation of the partials in $\mathrm{Co}-0.85$ at. $\% \mathrm{Fe}(\mathrm{hcp} \rightarrow \mathrm{dhcp})$ is similar to those observed in Co-5.75at ( $\mathrm{dhcp} \leftrightarrow \mathrm{fcc}$ ) [7]; in both cases there is an attractive interaction between the partials on neighbouring close packed planes. In the case of the dhcp $\leftrightarrow \mathrm{fcc}$ transformations paired partials are formed on adjacent planes (dipoles are forbidden on topological reasons since hexagonal symmetry is not preserved in this transformation). The paired partials further interact to compensate their long range strain fields. This requires three paired partials of different Burgers vectors (called a "tripole") that sum up to zero. Similar tripoles of partials were observed in single crystals of $\mathrm{CoNi}(\mathrm{hcp} \rightarrow \mathrm{fcc}$ ) [8] and shape memory alloys of $\mathrm{FeMnSiCrNi}(\mathrm{fcc} \rightarrow \mathrm{hcp}$ ) [9]. Atomic scale self accommodation involving dipoles and tripoles corresponds to transformation mode B; according to the scheme outlined by Christian [10] this mode is termed shuffle transformation since cancellation of the shears occurs over the smallest possible cell size. Contrary, transformation mode A involves partials of the same Burgers vector. In this case accommodation of the transformation shear strain was observed that occurs on an mesoscopic scale (selfcompensating shear transformation) leading to a surface relief [5].

\section{CONCLUSION}

The hcp $\rightarrow$ dhcp martensitic phase transformation was investigated in single crystals of Co- 0.85 at.\%Fe. The results obtained of both the TEM foils transformed by in-situ heating in the electron microscope and the specimens transformed in the bulk lead to a consistent picture of the transformation in agreement with transformation mode B. It is concluded that the partials at the fronts of the transformation form narrow dipoles compensating their long range strain fields on an atomic scale. Short range interactions might promote the nucleation of a correlated sequence of dipoles of a common Burgers vector. This leads to the formation of dhcp lamellae that contain minimum coherency strains.

\section{Acknowledgements}

Financial assistance from the Austrian FWF is acknowledged.

\section{References}

[1] Nishiyama Z., Martensitic Transformation, (Academic Press, New York, 1978)

[2] Onozuka T., Yamaguchi S., Hirabayashi H., Wakiyama T., J. Phys. Soc. Japan 37 (1974) 687-693

[3] Ishio S., Takahashi M., J. Magn. Magn. Mater. 46 (1984) 142-150

[4] Christian J. W., Martensite (American Society for Metals, Metals Park, Ohio, 1992) pp. 103-123

[5] Waitz T., Karnthaler H. P., Acta Mater., 45 (1997) 837-847

[6] Allen C. W., Liao K. C., Phys. Stat. Sol. (a), 74 (1982) 673-681

[7] Waitz T., Karnthaler H. P., Phil. Mag. A 73 (1996) 365-386

[8] Hitzenberger C., Karnthaler H. P., Phil. Mag. A, 64 (1991) 151-163

[9] Putaux J. L., Chevalier J. P., Acta Metall. Mater. 44 (1996) 1701-1716

[10] Christian J. W., J. Phys., C8, 5 (1995) 3-10 